\title{
Observation clinique
}

\section{Myxome des tissus mous : à propos d'un cas clinique}

\author{
Lamia Kissi, Chouaib Rifki, Ishane Ben Yahya* \\ Service d'0dontologie chirurgicale, Centre de consultations et de traitements dentaires, Faculté de Médecine dentaire, \\ Casablanca, Maroc
}

(Reçu le $1^{\text {er }}$ mars 2010, accepté le 15 mars 2010)

Mots clés :

myxome odontogénique / myxome des tissus mous / gencive

Key words: odontogenic myxoma / soft tissue myxoma / gingiva
Résumé - Le myxome des tissus mous ou myxome odontogène périphérique est classé dans les tumeurs bénignes des maxillaires. C'est une lésion rare, moins agressive que le myxome intra-osseux. Cliniquement et radiologiquement, il est difficile à différencier des autres lésions bénignes. Son diagnostic reste essentiellement histopathologique. Le traitement est conservateur et le taux de récidive faible. Les auteurs présentent un cas de myxome des tissus mous et détaillent les aspects cliniques, radiologiques et histopathologiques de cette lésion.

Abstract - Soft tissue myxoma: a case report. Myxoma of soft tissue or peripheral odontogenic myxoma is one of benign tumors of the jaws. It is a rare lesion that is less aggressive compared to its bone counterpart, and may be confused with other benign lesions. Its diagnosis therefore remains essentially histological. Treatment is conservative with a low recurrence rate.

The authors present a case of myxoma of soft tissue and detail the various clinical, radiological and histological findings of this lesion.
D'après la classification de l'OMS (1992), le myxome des maxillaires est une tumeur bénigne d'origine mésenchymateuse. Elle représenterait de 0,41 à $7,19 \%$ des tumeurs maxillaires [1-6].

Le myxome peut être de localisation centrale, c'est le myxome intra-osseux. Lorsqu'il est de localisation périphérique, on parle de myxome des tissus mous ou myxome odontogène périphérique, qui est beaucoup plus rare $[5,7]$.

Le but de ce travail est de mettre l'accent sur les particularités de cette lésion.

\section{Observation}

Une femme âgée de 42 ans, sans antécédents pathologiques particuliers, a consulté pour une tumeur mandibulaire, évoluant depuis 7 ans, occupant en partie le plancher buccal antérieur gauche.

L'examen exo-buccal a montré une symétrie faciale respectée et une absence d'adénopathies cervico-faciales. L'examen clinique endo-buccal a mis en évidence une masse gingivale siégeant sur le versant lingual de la crête alvéolaire édentée et s'étendant sur le plancher buccal adjacent. Le sommet de cette tumeur était ulcéré et portait l'empreinte des dents

\footnotetext{
^Correspondance : lamiakissi@yahoo.fr
}

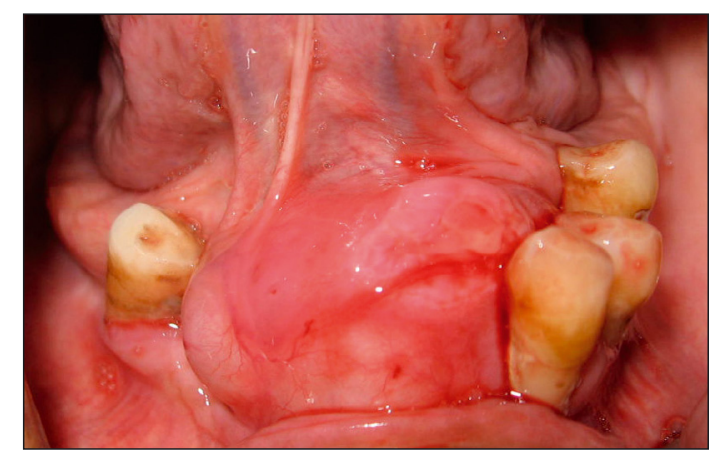

Fig. 1. Tumeur gingivale sur le plancher buccal antérieur gauche. Fig. 1. Gingival tumour on the left anterior oral floor.

antagonistes (Fig. 1). À la palpation, il s'agissait d'une tuméfaction ferme et indolore, les dents mandibulaires restantes étaient mobiles. La radiographie panoramique montrait une lyse osseuse en regard de la tumeur (Fig. 2). À ce stade, le diagnostic évoqué était celui d'épulis ou de fibrome ossifiant périphérique. L'exérèse de la tumeur a été réalisée sous anesthésie locale. La voie d'abord comportait une incision de la muqueuse à la partie supérieure de la masse, puis la lésion a été clivée pour obtenir son énucléation (Fig. 3). 


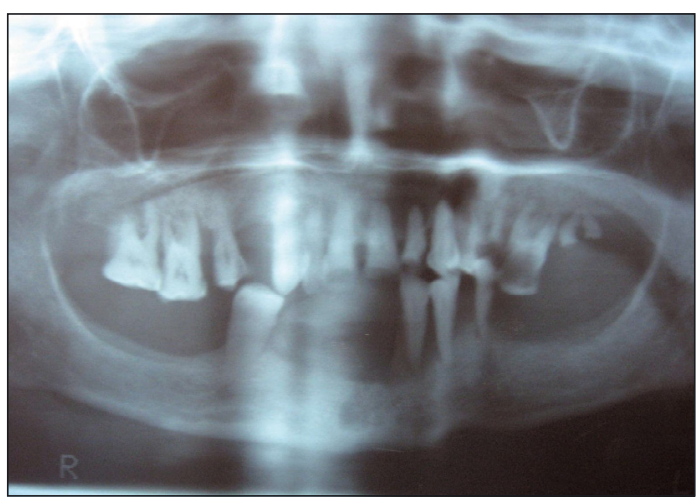

Fig. 2. Radiographie panoramique montrant la lyse osseuse. Fig. 2. Panoramic radiography showing bone lysis.

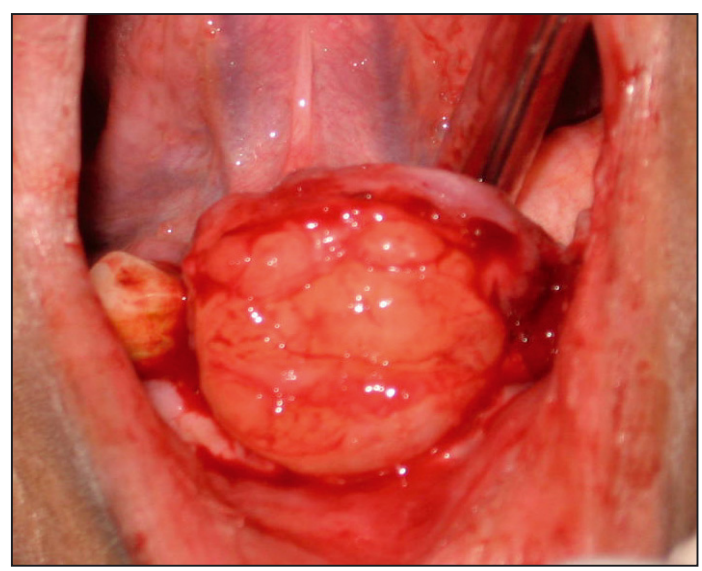

Fig. 3. Vue peropératoire : énucléation de la tumeur. Fig. 3. Peroperative view: enucleation of the tumour.

Les dents restantes ont été extraites et, après révision et curetage, une suture par un surjet passé a été réalisée.

La tumeur, de couleur blanc grisâtre et d'aspect gélatineux, était bien limitée (Fig. 4). L'examen anatomopathologique a montré qu'elle était constituée par un tissu fibreux richement vascularisé qui était le siège d'une prolifération fibroblastique, et comportait de larges foyers œdémateux et myxoïdes. Cet examen a conclu à un myxome des tissus mous.

Les suites opératoires ont été normales; la patiente ne s'est pas présentée à l'examen de contrôle.

\section{Commentaires}

Le myxome des maxillaires est une tumeur bénigne rare dont la pathogénie reste mal précisée. Il existe deux théories principales : la tumeur se développerait à partir des cellules souches persistant au sein du tissu conjonctif ou résulterait de la dégénérescence myxomateuse du stroma fibreux $[4-6,8]$. La présence occasionnelle d'épithélium odontogène au sein de la tumeur serait plus en faveur d'une origine dentaire, à partir de la papille folliculaire ou du ligament desmodontal [4].

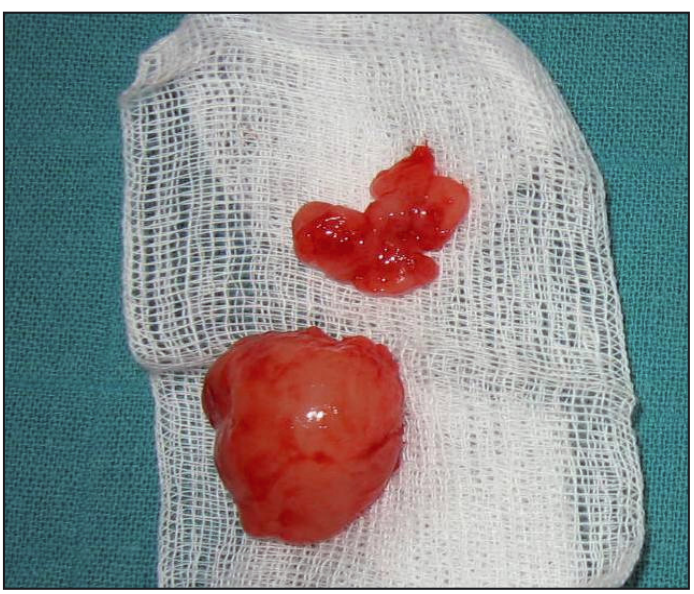

Fig. 4. Aspect macroscopique de la tumeur.

Fig. 4. Macroscopic view of the tumour.

Dans la sphère maxillo-faciale, deux formes sont décrites : le myxome intra-osseux et le myxome des tissus mous. Ce dernier se développe à partir de la gencive. Schmidseder et al. (1978) [9] classent tous les myxomes évoluant à partir de la crête alvéolaire, sans tenir compte de l'existence éventuelle d'une lyse osseuse, comme étant des myxomes périphériques. Le cas présenté semble donc correspondre au type périphérique.

Le myxome des tissus mous est plus rare que le myxome intra-osseux $[10,11]$. Une dizaine de cas ont été décrits dans la littérature. Il est généralement observé entre la deuxième et la quatrième décennie [4]. Il n'y a pas de sexe ratio prédominant, et la localisation mandibulaire est plus fréquente que la localisation maxillaire $[4,5]$. Cliniquement, la lésion se manifeste par une tuméfaction le plus souvent indolore, de croissance lente [12-14]. La palpation révèle une masse de consistance ferme, sans être dure. Les signes dentaires associés sont l'apparition d'une mobilité dentaire, le déplacement ou la perte de dents [6]. Dans le cas rapporté, la patiente présentait des mobilités dentaires mais le rôle de la tumeur était difficile à déterminer en raison de l'état bucco-dentaire médiocre.

L'examen radiologique standard peut montrer une ostéolyse mais elle reste moins importante que celle engendrée par le myxome intra-osseux $[5,9]$. La patiente présentait une discrète ostéolyse. Le CT-scan permet de préciser la topographie de la tumeur lorsqu'elle est étendue. Avec l'IRM, les myxomes ont un signal variable : un myxome des tissus mous donne un hypersignal en $\mathrm{T} 2$ et un hyposignal en $\mathrm{T} 1$; pour le myxome intra-osseux, c'est l'inverse [5,15].

L'aspect macroscopique est assez caractéristique : c'est une tumeur bien limitée, blanche, brillante, gélatiniforme, ayant à la palpation la consistance d'une balle de mousse $[6,16]$. L'aspect microscopique du myxome odontogène est caractérisé par la présence en petit nombre de cellules rondes ou anguleuses, disséminées dans un abondant stroma mucoïde. Il n'y a pas d'atypie cellulaire et l'activité mitotique est faible. Pour certains auteurs, la présence 
d'épithélium odontogène plaide en faveur de l'origine dentaire mais elle n'est pas nécessaire pour établir le diagnostic $[5,9,17]$. Dans le myxome des tissus mous, les cellules, beaucoup plus nombreuses, sont associées à du tissu fibreux [6] ; cet aspect est retrouvé dans le cas clinique présenté.

Le diagnostic différentiel fait évoquer d'abord une épulis, un granulome périphérique à cellules géantes, un lipome... [6].

Le traitement est chirurgical mais l'exérèse reste conservatrice car le myxome des tissus mous est bien limité et non invasif. Elle repose sur l'énucléation et le curetage soigneux $[5,6]$. Les myxomes périphériques ont un faible taux de récidive, probablement parce que la tumeur est bien limitée et qu'elle n'est pas agressive localement $[10,14]$, contrairement aux myxomes intra-osseux qui ont un taux élevé de récidive $[2,9]$.

\section{Conclusion}

Le myxome des tissus mous est une tumeur bénigne, rare par rapport au myxome intra-osseux mais il ne doit pas être méconnu. Les aspects cliniques et radiologiques sont insuffisants pour poser un diagnostic positif. Seul l'examen histopathologique permet le diagnostic de certitude.

\section{Références}

1. Kramer IRH, Pindborg JJ, Shear M. Histological typing of odontogenic tumors (p 23), $2^{\text {nd }}$ ed. Springer-Verlag, Berlin, 1992.

2. Lomuzio L, Nocini P, Favia G, Procaccini M, Mignogna MD. Odontogenic myxoma of the jaws: a clinical, radiologic, immunihistochemical and ultrastructural study. Oral Surg Oral Med Oral Pathol Oral Radiol Endod 1996;82:426-33.

3. Kaffe I, Naor H, Buchner A. Clinical and radiological features of odontogenic myxoma of the jaws. Dentomaxillofac Radiol 1997;26:299-303.
4. Naji A, Benjelloun A, Chekkoury-Idrissi A. Myxomes des maxillaries. www.courrierdudentiste 13 nov. 2009.

5. Gassab I, Hamroun A, Harrathi K, Ben Mahmoud F, Kadhi S, EL Kadhi F, Hafsa CH, Koubaa J, Gassab A. Myxome du maxillaire : à propos d'un cas. J Tun ORL 2007;18:43-5.

6. Escolle F, Gass M, Barriere P, Warter A, Feki A. À propos d'un myxome du maxillaire : difficultés diagnostiques et conduite à tenir. Med Buccale Chir Buccale 2005;11:23-9.

7. Nitassi S, Oujilal A, Boulaich A, Essakali L, Kzadri M. Les myxomes des maxillaires: à propos de 3 cas. Lettre Oto-rhinolaryngol Chir Cervicofac 2007;309-310:20-2.

8. Le Charpentier Y, Auriol M. Histopathologie bucco-dentaire et maxillo-faciale ( $p$ 88). Masson, Paris, 1997.

9. Shimoyama T, Horie N, Kato T, Tojo T, Nasu D, Kaneko T, Ide F. Soft tissue myxoma of the gingiva: report of a case and review of the literature of soft tissue myxoma in the oral region. $J$ Oral Sci 2000;42:107-9.

10. Frison L, Goudot P, Yachouh J. Tumeurs myxoïdes des tissus mous de la face. Rev Stomatol Chir Maxillofac 2010;111:21-4.

11. Ramarag PN, Shah SP. Peripheral myxoma of maxilla. A case report. Indian J Dent Res 2003;14:67-9.

12. Spencer KR, Smith A. Odontogenic myxoma: Case report with reconstructive considerations. Aust Dent J 1998;43:209-12.

13. Heymons $Q$, Nelissen $X$, Gilon $Y$, Jacquemin D, Fissette $J$. Myxome de la mandibule : à propos d'un cas. Rev Stomatol Chir Maxillofac 2002;103:239-41.

14. Aytac-Yazicioglu D, Eren H, Görgün S. Peripheral odontogenic myxoma located on the maxillary gingiva: report of a case and review of the literature. Oral Maxillofac Surg 2008;12:167-71.

15. Kawai T, Murakami S, Nishiyama H, Kishino M, Sakuda M, Fuchihata $H$. Diagnostic imaging for a case of maxillary myxoma with a review of the magnetic resonance images of myxoid lesion. Oral Surg Oral Med Oral Pathol Oral Radiol Endod 1997;84:449-54.

16. Essakalli HL, Lazrak A, Benchaqroun L, Jazouli N, Kzadri M, El Amarti A. Myxome du maxillaire supérieur : à propos d'un cas. Rev Stomatol Chir Maxillofac 1996;97:117-20.

17. Chang SH, Lee KF, Chan CP, Kuo SB. Myxoma of the gingiva. A case report and literature review. Chang Gung Med J 2001;24:826-31. 